\title{
Association of $\gamma$-glutamyl transferase with premature coronary artery disease
}

\author{
MADANKUMAR GHATGE ${ }^{1,2}$, ANKIT SHARMA ${ }^{1-3}$ and RAJANI KANTH VANGALA ${ }^{1,3}$ \\ ${ }^{1}$ Tata Proteomics and Coagulation Unit, Thrombosis Research Institute, Narayana Hrudayalaya Hospital, \\ Anekal Taluk, Bangalore 560099; ${ }^{2}$ Manipal University, Madhav Nagar, Manipal, Karnataka 576104; \\ ${ }^{3}$ Elizabeth and Emmanuel Kaye Bioinformatics and Biostatistics Unit, Thrombosis Research Institute, \\ Narayana Hrudayalaya Hospital, Anekal Taluk, Bangalore 560099, India
}

Received September 24, 2015; Accepted December 7, 2015

DOI: $10.3892 /$ br. 2016.576

\begin{abstract}
Accumulating evidence from epidemiological studies suggests that higher $\gamma$-glutamyl transferase (GGT) levels in the blood are associated with the incident of cardiovascular disease (CVD), including atherosclerosis, and have prognostic importance. However, to the best of our knowledge, the association of the GGT level with premature coronary artery disease (CAD) in an Asian Indian population has not been evaluated. In the present study, 240 (120 unaffected and 120 CAD affected) young subjects (males, $\leq 45$ years and females, $\leq 50$ years) were selected. The markers assayed were GGT, high-sensitivity C-reactive protein, lipids, secretory phospholipase A2, neopterin, myeloperoxidase, interleukin-6, cystatin-C, tumor necrosis factor-like weak inducer of apoptosis and lipoprotein (a). The plasma GGT levels in these subjects showed a positive correlation with quantitative variables, such as waist circumference, triglycerides, neopterin levels and cross-sectional correlation with qualitative variable smoking. The findings suggest that the subjects in the highest tertile of GGT had a 2.1-fold [odds ratio (OR), 2.104; 95\% confidence interval (CI), 1.063-4.165; $\mathrm{P}=0.033$ ] higher risk of developing premature $\mathrm{CAD}$ in comparison with the reference tertile. Furthermore, a $1 \mathrm{U} / 1$ increase of GGT (on a log scale) increased the OR by 5.2-fold (OR, 5.208; 95\% CI, 1.018-24.624; $\mathrm{P}=0.048)$ and 7.4-fold (OR, 7.492; 95\% CI, 1.221-45.979; $\mathrm{P}=0.030)$ on addition of associated risk factors. In conclusion, the elevated plasma GGT levels potentially indicate increased oxidative stress and the risk of developing premature CAD. Therefore, these findings could be potentially used in the risk stratification of premature CAD following further evaluation.
\end{abstract}

Correspondence to: Dr Rajani Kanth Vangala, Tata Proteomics and Coagulation Unit, Thrombosis Research Institute, Narayana Hrudayalaya Hospital, 258/A Bommasandra Industrial Area, Anekal Taluk, Bangalore 560099, India

E-mail: rajani@triindia.org.in

Key words: premature coronary artery disease, $\gamma$-glutamyl transferase, oxidative stress, reactive oxygen species, risk stratification

\section{Introduction}

Coronary artery disease (CAD) has become a leading cause of morbidity and mortality in India and worldwide $(1,2)$. In India, CAD affects younger people (aged $<45$ years) with a higher severity resulting in a 4-fold higher incidence compared to the western population. The present day preventive strategies mainly depend on the conventional risk factors, such as diabetes, hypertension, obesity and levels of lipids [including total cholesterol, triglycerides, high-density lipoprotein (HDL) and low-density lipoprotein (LDL)]. However, a number of supposedly healthy people without the majority of these risk factors develop the disease $(3,4)$. This high prevalence of CAD in Asian Indians accentuates the requirement for identification of biomarkers for an improved risk stratification $(5,6)$.

Serum $\gamma$-glutamyl transferase (GGT) levels, a traditional marker of hepatobiliary dysfunction, are positively associated with atherosclerosis and have prognostic importance. An increase of $1 \mathrm{U} / \mathrm{l}$ of GGT (on a log scale) was found to increase the risk of coronary heart disease (CHD) by 20\%, stroke by $54 \%$ and the combined risk of stroke and CHD by $34 \%$ (7). Furthermore, correlation of GGT has been found with cardiovascular risk factors, such as hypertension, stroke, diabetes, dyslipidemia and metabolic syndrome (8). The presence of catalytically active GGT molecules in the atheromatous plaque also reinforce the association of GGT with atherosclerosis (9). However, the association of GGT to premature CAD in Asian Indians has not yet been evaluated. The present study was undertaken to investigate the association of plasma GGT levels with CAD in young Asian Indians and its interaction with other biomarkers for potential use in an improved risk stratification.

\section{Materials and methods}

Subjects. A total of 2,318 subjects were enrolled in the phase-I of the Indian Atherosclerosis Research Study (IARS) (10) between 2004-2006, which is a prospective study designed according to the principles and the guidelines of World Medical Association Declaration of Helsinki and the Indian Council of Medical Research and approved by Thrombosis Research Institute Ethics committee. Signed informed consent 
was obtained from all the participants who were enrolled, and 240 age- and gender-matched subjects (120 unaffected and 120 affected) were selected for this study. All the subjects were $\leq 45$ years of age for males and $\leq 50$ years for females at the onset of the disease. The individuals with an abnormal electrocardiogram (ECG), positive angiogram showing $>70 \%$ stenosis in any one of the coronary arteries or $>50 \%$ in two or more arteries and individuals posted for coronary artery bypass graft $(\mathrm{CABG})$ surgery or percutaneous coronary intervention were considered as CAD subjects. The unaffected subjects were all healthy volunteers without clinical signs of CAD, as confirmed by ECG. None of the participants enrolled in the study had inflammatory disorders, concomitant infections or any other major illness such as cancer, liver or renal disease.

Telephonic assessment of cardiac health status. Periodic telephonic follow-up of all the study participants was undertaken to obtain an update on the cardiac health status, and five rounds of follow-up for an average time of 18 months were completed. Endpoints included non-fatal events (re-do percutaneous transluminal coronary angioplasty/repeat CABG/heart attack or stroke) and fatal events (fatal heart attack/fatal stroke/sudden death or cardiac arrest). This information was verified through either paper trail or hospital records, wherever available.

Assessment of risk factors. All the participants were assessed for the presence of the risk factors by personal interviews, which included demographics (age, gender, occupation and socio-economic status), lifestyle, health characteristics (diet, waist circumference, smoking status and use of alcohol), and medical history [hypertension and diabetes, systolic and diastolic blood pressure, LDL and HDL-cholesterol (HDL-c), and triglycerides].

Assessment of lipids. Venous blood samples $(20 \mathrm{ml})$ were collected after overnight fasting (12 h). The samples were centrifuged at $2,000 \mathrm{x} \mathrm{g}$ for $20 \mathrm{~min}$ at $25^{\circ} \mathrm{C}$, following which $1 \mathrm{ml}$ aliquots of serum and plasma were stored at $-80^{\circ} \mathrm{C}$. Total cholesterol and triglycerides were measured using reagents and standards from Randox Laboratories (Antrim, UK) in a Cobas-Fara II Clinical Chemistry auto-analyzer (Hoffman La Roche Ltd., Basel, Switzerland). For the estimation of HDL-c, the non-HDL fractions were precipitated with a mixture of $2.4 \mathrm{mmol} / \mathrm{l}$ phosphotungstic acid and $39 \mathrm{mmol} / \mathrm{l}$ magnesium chloride (Bayer Diagnostics, Baroda, Gujarat, India) prior to estimation. The LDL concentration was calculated using the Friedewald formula. The inter-assay coefficient of variation for commercial controls and normal human pooled serum/plasma (NHP) was 4.9-7.0\% for total cholesterol, 6.1-7.7\% for triglycerides, and 7.1-12.2\% for HDL-c.

Biomarker evaluation. All the plasma or serum biomarkers were measured by ELISA (enzyme-linked immunosorbent assay) or the immunoturbidometric method. Plasma GGT and high-sensitivity C-reactive protein (hsCRP) concentrations were measured using a Flex reagent cartridge in Dimension

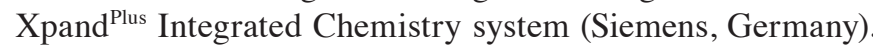
Lipoprotein (a) [lp(a)] (Randox Laboratories) was measured in the Cobas-Fara II Clinical Chemistry auto-analyzer. Plasma levels of interleukin-6 (IL-6), cystatin C (R\&D systems, Minneapolis, MN, USA), neopterin (IBL International, Atlanta, GA, USA), myeloperoxidase (MPO) (Mercodia, Uppasala, Sweden) and tumor necrosis factor-like weak inducer of apoptosis (TWEAK) (eBiosciences, Vienna, Austria) were measured using ELISA kits. The secretory phospholipase A2 (sPLA2) was measured by a sandwich immunometric assay (Cayman Corporation, Ann Arbor, MI, USA). The inter-assay coefficient of variation of in-house controls (NHP) was $4.40 \%$ for GGT, 5.2\% for lp(a), 4.3\% for IL-6, 5.37\% for sPLA2, 7.85\% for hsCRP, 5.99 to $11.8 \%$ for neopterin, $11.8 \%$ for cystatin C, $9.3 \%$ for myeloperoxidase, and $7 \%$ for TWEAK.

Statistical analysis. The variations in the baseline characteristics between the unaffected and affected subjects were studied using the Student's t-test and univariate analysis of variance for quantitative variables. The quantitative parameters were adjusted for age, gender, hypertension, diabetes mellitus and smoking. $\chi^{2}$ test was used to assess the association of discrete outcomes with premature CAD. Results are represented as mean \pm standard error for quantitative variables, whereas the discrete outcomes were represented as frequencies. The Shapiro-Wilk and Kolmogorove-Smirnov test were used to assess the normality of the data. The data with skewed distribution were natural log-transformed. The association of GGT with lipids, inflammatory and oxidative stress markers was evaluated by Pearson's correlation for quantitative variables and partial correlation was used for conventional risk factors (CRFs; which were age, gender, hypertension diabetes mellitus and smoking) adjusted analysis. The association of GGT with discrete outcomes was evaluated using $\chi^{2}$ test.

In order to review the association between higher levels of GGT and incidence of premature CAD, GGT levels were categorized in to tertiles. The odds ratios (OR) with 95\% confidence interval (CI) were obtained by comparing top tertiles, considering the first tertile as the reference category using logistic regression analysis. The logistic regression analysis was also used to build different models with multiple markers having GGT in common. The discriminative ability of a significant model with the highest OR was evaluated using receiver operating characteristic analysis. The significance of improvement in the area under the curve (AUC) between CRF and GGT + CRF models was assessed using DeLong's test. A two-tailed P-value $<0.05 \%$ was considered to indicate a statistically significant difference. All the above statistical analyses were carried out using statistical package for social sciences (SPSS) version 17.0 (SPSS, Inc., Chicago, IL, USA).

\section{Results}

Baseline clinical characteristics of unaffected subjects and premature $C A D$ patients. The clinical characteristics of the 240 age- and gender-matched study subjects (120 affected and 120 unaffected) are provided in Table I. The CAD-affected subjects showed a high frequency of hypertension and diabetes, and low levels of HDL-c compared to the unaffected subjects. The frequency of smokers was also significantly higher in the CAD-affected group. The affected subjects showed a lower level of LDL compared to the unaffected, possibly due to statin usage in this group. Six out of 9 protein markers assayed in the 
Table I. Baseline characteristics of the study population.

\begin{tabular}{|c|c|c|c|}
\hline Variables & Unaffected, $\mathrm{n}=120$ & Affected, $n=120$ & P-value \\
\hline Age, years & $41.78 \pm 0.40$ & $41.79 \pm 0.40$ & 0.988 \\
\hline Age at onset, years & - & $39.50 \pm 0.43$ & - \\
\hline Waist circumference, $\mathrm{cm}$ & $89.57 \pm 0.85$ & $88.41 \pm 0.87$ & 0.341 \\
\hline \multicolumn{4}{|l|}{ Gender, n (\%) } \\
\hline Males & $93(77.5)$ & $93(77.5)$ & 1.00 \\
\hline Females & $27(22.5)$ & $27(22.5)$ & \\
\hline Smoking, n (\%) & $30(25.0)$ & $55(45.8)$ & 0.001 \\
\hline Alcohol habit, n (\%) & $24(20.0)$ & $16(13.3)$ & 0.053 \\
\hline Hypertension, n (\%) & $19(15.8)$ & $45(37.5)$ & $<0.001$ \\
\hline Diabetes, n (\%) & $16(13.3)$ & $32(26.7)$ & 0.007 \\
\hline Total cholesterol, mg/dl & $181.83 \pm 3.94$ & $154.00 \pm 3.45$ & $<0.001$ \\
\hline Triglyceride, mg/dl & $150.20 \pm 8.30$ & $163.17 \pm 6.26$ & 0.152 \\
\hline High-density lipoprotein, mg/dl & $39.00 \pm 0.88$ & $35.97 \pm 0.76$ & 0.010 \\
\hline Low-density lipoprotein, mg/dl & $112.78 \pm 3.36$ & $85.35 \pm 3.14$ & $<0.001$ \\
\hline$\gamma$-glutamyl transferase, $U / 1$ & $30.94 \pm 0.90$ & $34.48 \pm 1.00$ & 0.009 \\
\hline Secretory phospholipase A2, pg/ml & $2,518.02 \pm 130.67$ & $2,720.00 \pm 130.15$ & 0.027 \\
\hline Lipoprotein (a), mg/dl & $18.70 \pm 1.55$ & $27.20 \pm 2.34$ & 0.019 \\
\hline Myeloperoxidase, $\mu \mathrm{g} / 1$ & $304.61 \pm 14.73$ & $356.51 \pm 19.89$ & 0.037 \\
\hline Neopterin, nmol/l & $7.65 \pm 0.32$ & $8.50 \pm 0.34$ & 0.034 \\
\hline Interleukin-6, pg/ml & $2.98 \pm 0.15$ & $3.66 \pm 0.19$ & 0.006 \\
\hline Cystatin C, ng/ml & $1,053.61 \pm 30.43$ & $1,058.03 \pm 32.74$ & 0.899 \\
\hline High-sensitivity C-reactive protein, mg/dl & $2.35 \pm 0.18$ & $2.32 \pm 0.19$ & 0.599 \\
\hline Tumor necrosis factor-like weak inducer of apoptosis, $\mathrm{pg} / \mathrm{ml}$ & $355.94 \pm 30.95$ & $347.00 \pm 17.99$ & 0.802 \\
\hline Statins, n (\%) & $5(4.2)$ & $82(68.3)$ & $<0.001$ \\
\hline
\end{tabular}

present study were significantly different between the affected and unaffected subjects. As shown in Table I, the mean GGT levels were significantly higher in CAD-affected subjects in comparison with the unaffected subjects $(34.48 \pm 1.00$ vs. $30.94 \pm 0.90 \mathrm{U} / 1 ; \mathrm{P}=0.009)$ and similar results were also obtained for $\mathrm{lp}$ (a) $(27.20 \pm 2.34$ vs. $18.70 \pm 1.55 \mathrm{mg} / \mathrm{dl}$; $\mathrm{P}=0.019)$.

Correlation of GGT levels with inflammatory and lipid markers. The GGT levels showed a significant moderate-positive correlation with the oxidative stress marker neopterin, and remained borderline significant following adjustment for the age, gender, hypertension, diabetes mellitus and smoking (Table II). GGT was also positively correlated to triglyceride levels and waist circumference, and remained significant following adjustment with age, gender, hypertension, diabetes mellitus and smoking (all the significant correlation coefficients were $<0.3$ ).

Cross-sectional correlation was carried out to understand the association between GGT and binary outcomes. The present analysis did not observe any association of hypertension, diabetes and alcohol habit with GGT levels (Table III). The number of smokers in the second and third tertile of GGT was higher in comparison to the first, and there was a positive association between GGT levels and smoking.
Association of GGT tertiles with CAD. In comparison to the low GGT level of $<22.59 \mathrm{U} / 1$ reference group, the high GGT levels of $\geq 32.21 \mathrm{U} / 1$ (third tertile) showed an OR of 1.909 (95\% CI, 1.036-3.517; $\mathrm{P}=0.038$ ). Following adjustment to the GGT-associated risk factors and CAD risk factors, the OR increased to 2.104 (95\% CI, 1.063-4.165; P=0.033), i.e., the subjects with GGT levels of $\geq 32.21 \mathrm{U} / 1$ had a 2.11 -fold higher risk of premature CAD in comparison to $<22.59 \mathrm{U} / 1$. The intermediate GGT levels (second tertile) group did not show any association with premature CAD prior and subsequent to adjustment to the conventional risk factors (Table IV).

Risk association assessment. The logistic regression model was used to assess the risk predictive ability of GGT alone and along with lipid, inflammatory, oxidative stress markers and conventional risk factors. GGT alone was found to have an OR of 4.94 (95\% CI, 1.015-24.095; P=0.048), which improved to 5.208 (95\% CI, 1.018-24.624; $\mathrm{P}=0.048)$ upon adjusting for the conventional risk factors. The OR of this model further improved to 5.64 (95\% CI, 1.004-31.642; $\mathrm{P}=0.049)$ upon addition of $\operatorname{lp}(\mathrm{a})$. The above combination of GGT and lp(a) exhibited a higher OR of 7.492 (95\% CI, 1.221-45.979; $\mathrm{P}=0.030$ ) (Table V) following adjustment for CRFs. 
Table II. Correlation of $\gamma$-glutamyl transferase (GGT) levels with oxidative stress, inflammatory markers and lipid markers.

\begin{tabular}{|c|c|c|c|c|}
\hline \multirow[b]{2}{*}{ Characteristics } & \multicolumn{2}{|c|}{ Unadjusted } & \multicolumn{2}{|c|}{ Adjusted } \\
\hline & Correlation & P-value & Correlation & P-value \\
\hline Lipoprotein (a), mg/dl & 0.050 & 0.465 & 0.050 & 0.480 \\
\hline Secretory phospholipase A2, pg/ml & 0.036 & 0.584 & 0.092 & 0.165 \\
\hline Interleukin- $6, \mathrm{pg} / \mathrm{ml}$ & 0.090 & 0.164 & 0.093 & 0.161 \\
\hline Myeloperoxidase, $\mu \mathrm{g} / 1$ & 0.106 & 0.102 & 0.068 & 0.310 \\
\hline Neopterin, $\mathrm{nmol} / \mathrm{l}$ & 0.172 & 0.008 & 0.127 & 0.050 \\
\hline Total cholesterol, mg/dl & 0.110 & 0.090 & 0.136 & 0.041 \\
\hline High-density lipoprotein, mg/dl & -0.035 & 0.587 & 0.012 & 0.862 \\
\hline Low-density lipoprotein, mg/dl & 0.001 & 0.993 & 0.043 & 0.519 \\
\hline Triglyceride, $\mathrm{mg} / \mathrm{dl}$ & 0.292 & $<0.001$ & 0.168 & 0.011 \\
\hline Waist circumference, $\mathrm{cm}$ & 0.252 & $<0.001$ & 0.251 & $<0.001$ \\
\hline
\end{tabular}

Partial correlations adjusted for age, gender, hypertension, diabetes mellitus and smoking status.

Table III. Cross-sectional correlation of binary outcomes with $\gamma$-glutamyl transferase (GGT).

\begin{tabular}{lccrr}
\hline & \multicolumn{4}{c}{ GGT tertiles } \\
\cline { 2 - 4 } Characteristics & $\begin{array}{c}\text { Tertile 1 } \\
(<22.59 \mathrm{U} / \mathrm{l})(\mathrm{n}=86)\end{array}$ & $\begin{array}{c}\text { Tertile 2 } \\
(22.59-32.21 \mathrm{U} / \mathrm{l})(\mathrm{n}=71)\end{array}$ & $\begin{array}{c}\text { Tertile 3 } \\
(\geq 32.21 \mathrm{U} / \mathrm{l})(\mathrm{n}=83)\end{array}$ & P-value \\
\hline Smoking, $\mathrm{n}(\%)$ & $19(22.1)$ & $26(36.6)$ & $40(48.2)$ & 0.002 \\
Hypertension, $\mathrm{n}(\%)$ & $17(19.8)$ & $22(31.0)$ & $25(30.1)$ & 0.194 \\
Diabetes melitus, $\mathrm{n}(\%)$ & $14(16.3)$ & $15(21.1)$ & $19(22.9)$ & 0.539 \\
Alcohol habits, $\mathrm{n}(\%)$ & $10(11.6)$ & $14(19.7)$ & $16(19.3)$ & 0.293 \\
\hline
\end{tabular}

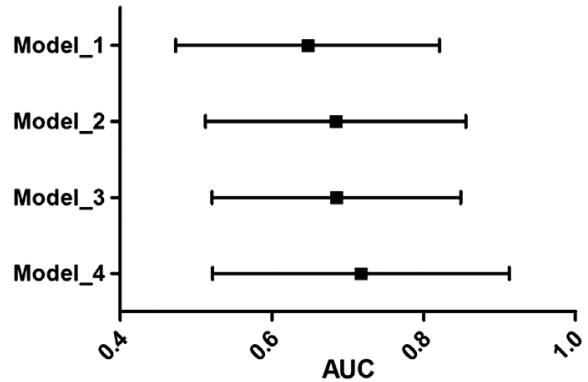

Figure 1. AUC of four different models with corresponding confidence intervals. Model 1, GGT alone; model 2, conventional risk factors (age, gender, smoking, hypertension and diabetes melitus); model 3, GGT $+\mathrm{lp}(\mathrm{a})$; model 4 , GGT + lp(a) + conventional risk factors. AUC, area under the curve; GGT, $\gamma$-glutamyl transferase; $\operatorname{lp}(\mathrm{a})$, lipoprotein (a).

The addition of MPO to the GGT was significant with a reduction in the OR of GGT from 4.94 to 3.48; however, the significance of the model was lost following adjustment with CRFs (Table V). Other inflammatory and oxidative stress markers did not add any significant value to the predictive ability of the GGT.

Discriminative ability of the model. The C-statistic analysis was performed to assess the discriminative ability of the models built using logistic regression (Fig. 1). The discriminative ability of GGT alone was tested and compared with the other models. The GGT alone significantly classified the $61.5 \%$ population correctly. However, conventional risk factors used in the model building exhibited an AUC of 0.647 (95\% CI, 0.577-0.717; P<0.001). The GGT and $\mathrm{lp}(\mathrm{a})$ together increased the AUC to 0.654 (95\% CI, 0.576-0.732; $\mathrm{P}<0.001$ ). A combined model of GGT and $\mathrm{lp}$ (a) plus conventional risk factors showed the best performance with an AUC of 0.711 (95\% CI, 0.642-0.799; P<0.001). The DeLong's test indicated that the improvement of AUC from CRF to biomarkers [GGT $+1 \mathrm{p}(\mathrm{a})]$ adjusted to CRFs was significant $(\mathrm{P}<0.001)$. All the models built were tested for best-fit using the Hosmer-Lemeshow test.

\section{Discussion}

The key outcomes of the present study were two-fold. The first section of the results suggest that increasing GGT levels were associated with the CAD risk factors, such as smoking, hyperlipidemia, waist circumference and oxidative stress marker neopterin, indicating a possible existence of a condition such as oxidative stress in the subjects. The second section of the results indicates that higher levels of GGT in the blood are associated with an increased risk of premature CAD. However, 
Table IV. Risk of coronary artery disease events based on $\gamma$-glutamyl transferase (GGT) tertiles.

\begin{tabular}{|c|c|c|c|c|}
\hline \multirow[b]{2}{*}{ GGT tertiles } & \multicolumn{2}{|c|}{ Unadjusted } & \multicolumn{2}{|c|}{ Adjusted } \\
\hline & OR $(95 \% \mathrm{CI})$ & P-value & OR (95\% CI) & P-value \\
\hline Tertile 1 & - & - & - & - \\
\hline Tertile 2 & $1.212(0.647-2.289)$ & 0.542 & $1.057(0.528-2.117)$ & 0.875 \\
\hline Tertile 3 & 1.909 (1.036-3.517) & 0.038 & $2.104(1.063-4.165)$ & 0.033 \\
\hline
\end{tabular}

Adjusted for age, gender, hypertension, diabetes mellitus, smoking status and baseline lipoprotein (a) levels. OR, odds ratio; CI, confidence interval.

Table V. Association of $\gamma$-glutamyl transferase (GGT) levels with coronary artery disease in the presence of lipid, inflammatory and oxidative stress markers.

\begin{tabular}{|c|c|c|c|c|}
\hline \multirow[b]{2}{*}{ Model } & \multicolumn{2}{|c|}{ Unadjusted } & \multicolumn{2}{|c|}{ Adjusted } \\
\hline & OR $(95 \% \mathrm{CI})$ & P-value & OR $(95 \% \mathrm{CI})$ & P-value \\
\hline \multicolumn{5}{|l|}{ Lipid and inflammatory markers } \\
\hline GGT & $4.944(1.015-24.095)$ & 0.048 & $5.208(1.018-24.624)$ & 0.048 \\
\hline +Lipoprotein (a) & $5.636(1.004-31.642)$ & 0.049 & $7.492(1.221-45.979)$ & 0.030 \\
\hline +Secretory phospholipase A2 & $4.829(0.972-23.980)$ & 0.054 & $5.138(0.952-27.742)$ & 0.057 \\
\hline+ Interleukin-6 & $4.445(0.972-22.011)$ & 0.068 & $4.691(0.866-25.339)$ & 0.073 \\
\hline \multicolumn{5}{|l|}{ Oxidative stress markers } \\
\hline +Myeloperoxidase & $3.485(1.199-10.128)$ & 0.022 & $4.555(0.849-24.443)$ & 0.077 \\
\hline+ Neopterin & $3.816(0.746-19.060)$ & 0.103 & $4.041(0.741-21.923)$ & 0.105 \\
\hline
\end{tabular}

Adjusted for age, gender, hypertension, diabetes mellitus and smoking status. OR, odds ratio; CI, confidence interval.

the other major risk factors, such as diabetes mellitus, hypertension and dyslipidemia, were not associated with GGT levels but were significantly associated with the premature CAD. Therefore, we adjusted the GGT levels with these risk factors and the association remained significant following adjustment. This suggests that increased GGT levels could act as an independent factor in the process of premature CAD development in the Indian population.

Previously a number of studies have shown the association of increased GGT levels with CAD risk factors and CAD development (11-14); however, to best of our knowledge there are no studies showing the association of increased GGT levels with premature CAD in the Indian population. In the present study, a pilot investigation was performed to show this possible association between premature CAD and GGT levels. The findings indicate that young subjects in the highest tertile have a 2.1-fold higher risk with respect to reference tertile (Table IV) and a unit increase of GGT levels (on a log scale) increases the risk of premature CAD by 5-fold, which increased to 7-fold in the presence of higher $1 p(a)$ levels (Table V).

The results of the present study indicate the association of GGT levels with premature CAD, which was independent of alcohol habits. However, the correlation between GGT, smoking, triglycerides, waist circumference and neopterin levels indicates a potential mechanism in the development of the disease. Experimental evidence suggests that GGT, by regulating intracellular glutathione, acts as an antioxidant $(15,16)$. Whereas GGT has been shown to have a prooxidant role by producing reactive oxygen species in the presence of transition metal ions (such as iron) (17-19), which can lead to endothelial dysfunction. However, this increased deposition of iron could be due to smoking and may contribute to the endothelial dysfunction (20). In addition, our previous study also revealed reduced adiponectin levels in CAD subjects (21). Kozakova et al (22) reported the importance of GGT linking fatty liver condition and atherosclerosis potentially by regulating the adiponectin level. In accordance with these findings, the present data suggests that in a fatty liver condition, higher GGT levels may indicate the migration of aortic SMC into the intimal layer, and hence, inducing the atherosclerotic condition. In conclusion, elevated plasma GGT levels may indicate an increased risk of developing premature CAD.

There were strengths and limitations of the present study. The present study was carried out on a relatively small sample size, and requires further validation on larger subjects. However, this preliminary data revealed GGT as one of the risk factors and biomarkers in premature CAD subjects. GGT is a low cost and readily available assay, thus, validating in a larger cohort and translating the findings into clinical practice would be comparatively easy. 


\section{Acknowledgements}

The authors are grateful for the support of the funding agencies: Department of Biotechnology, Ministry of Science and Technology, Government of India (grant no. BT/01/CDE/08/07), the Tata Social Welfare Trust, India (grant no. TSWT/IG/SNB/JP/Sdm), Garfield Weston Foundation, UK, Foundation Bay, UK and Bharati Foundation, India. The authors thank all the investigators, staff, and administrative teams and participants of IARS from Narayana Hrudayalaya, Bangalore and Asian Heart Centre (Mumbai) for their contribution. The authors are grateful to the patients and their family members for participating in the study.

\section{References}

1. Kanjilal S, Rao VS, Mukherjee M, Natesha BK, Renuka KS Sibi K, Iyengar SS and Kakkar VV: Application of cardiovascular disease risk prediction models and the relevance of novel biomarkers to risk stratification in Asian Indians. Vasc Health Risk Manag 4: 199-211, 2008.

2. Mathers CD and Loncar D: Projections of global mortality and burden of disease from 2002 to 2030. PLoS Med 3: e442, 2006.

3. Greenland P, Knoll MD, Stamler J, Neaton JD, Dyer AR, Garside DB and Wilson PW: Major risk factors as antecedents of fatal and nonfatal coronary heart disease events. JAMA 290 891-897, 2003.

4. Khot UN, Khot MB, Bajzer CT, Sapp SK, Ohman EM, Brener SJ, Ellis SG, Lincoff AM and Topol EJ: Prevalence of conventional risk factors in patients with coronary heart disease. JAMA 290: 898-904, 2003

5. Goyal A and Yusuf S: The burden of cardiovascular disease in the Indian subcontinent. Indian J Med Res 124: 235-244, 2006.

6. Sharma M and Ganguly NK: Premature coronary artery disease in Indians and its associated risk factors. Vasc Health Risk Manag 1: 217-225, 2005.

7. Atar AI, Yilmaz OC, Akin K, Selcoki Y, Er O and Eryonucu B: Association between gamma-glutamyltransferase and coronary artery calcification. Int J Cardiol 167: 1264-1267, 2013.

8. Poelzl G, Eberl C, Achrainer H, Doerler J, Pachinger O, Frick M and Ulmer H: Prevalence and prognostic significance of elevated gamma-glutamyltransferase in chronic heart failure. Circ Heart Fail 2: 294-302, 2009.

9. Paolicchi A, Emdin M, Ghliozeni E, Ciancia E, Passino C, Popoff $\mathrm{G}$ and Pompella A: Images in cardiovascular medicine. Human atherosclerotic plaques contain gamma-glutamyl transpeptidase enzyme activity. Circulation 109: 1440, 2004
10. Shanker J, Maitra A, Rao VS, Mundkur L, Dhanalakshmi B, Hebbagodi S and Kakkar VV: Rationale, design \& preliminary findings of the Indian atherosclerosis research study. Indian Heart J 62: 286-295, 2010.

11. Dhingra R, Gona P, Wang TJ, Fox CS, D'Agostino RB Sr and Vasan RS: Serum gamma-glutamyl transferase and risk of heart failure in the community. Arterioscler Thromb Vasc Biol 30: 1855-1860, 2010

12. Emdin M, Passino C, Michelassi C, Donato L, Pompella A and Paolicchi A: Additive prognostic value of gamma-glutamyltransferase in coronary artery disease. Int J Cardiol 136: 80-85, 2009.

13. Ruttmann E, Brant LJ, Concin H, Diem G, Rapp K and Ulmer H; Vorarlberg Health Monitoring and Promotion Program Study Group: Gamma-glutamyltransferase as a risk factor for cardiovascular disease mortality: An epidemiological investigation in a cohort of 163,944 Austrian adults. Circulation 112: 2130-2137, 2005.

14. Wannamethee G, Ebrahim $S$ and Shaper AG: Gamma-glutamyltransferase: Determinants and association with mortality from ischemic heart disease and all causes. Am J Epidemiol 142: 699-708, 1995.

15. Karp DR, Shimooku K and Lipsky PE: Expression of gamma-glutamyl transpeptidase protects ramos B cells from oxidation-induced cell death. J Biol Chem 276: 3798-3804, 2001

16. Takahashi Y, Oakes SM, Williams MC, Takahashi S, Miura T and Joyce-Brady M: Nitrogen dioxide exposure activates gamma-glutamyl transferase gene expression in rat lung. Toxicol Appl Pharmacol 143: 388-396, 1997.

17. Paolicchi A, Tongiani R, Tonarelli P, Comporti $\mathrm{M}$ and Pompella A: gamma-Glutamyl transpeptidase-dependent lipid peroxidation in isolated hepatocytes and HepG2 hepatoma cells. Free Radic Biol Med 22: 853-860, 1997.

18. Stark AA: Oxidative metabolism of glutathione by gamma-glutamyl transpeptidase and peroxisome proliferation: The relevance to hepatocarcinogenesis. A hypothesis. Mutagenesis 6: 241-245, 1991.

19. Stark AA, Russell JJ, Langenbach R, Pagano DA, Zeiger E and Huberman E: Localization of oxidative damage by a glutathione-gamma-glutamyl transpeptidase system in preneoplastic lesions in sections of livers from carcinogen-treated rats. Carcinogenesis 15: 343-348, 1994.

20. Fukami K, Yamagishi S, Iida S, Matsuoka H and Okuda S: Involvement of iron-evoked oxidative stress in smoking-related endothelial dysfunction in healthy young men. PLoS One 9: e89433, 2014

21. Shanker J, Rao VS, Ravindran V, Dhanalakshmi B, Hebbagodi S and Kakkar VV: Relationship of adiponectin and leptin to coronary artery disease, classical cardiovascular risk factors and atherothrombotic biomarkers in the IARS cohort. Thromb Haemost 108: 769-780, 2012

22. Kozakova M, Palombo C, Eng MP, Dekker J, Flyvbjerg A, Mitrakou A, Gastaldelli A and Ferrannini E; RISC Investigators: Fatty liver index, gamma-glutamyltransferase and early carotid plaques. Hepatology 55: 1406-1415, 2012. 